\title{
A importância da qualidade da informação na predição de valores genéticos para características de crescimento em bovinos da raça Nelore
}

\author{
[The importance of quality information to predict breeding values for growth traits in Nelore cattle] \\ C. Pereira ${ }^{1}$, C.U. Faria ${ }^{1}$, R. Lôbo ${ }^{2}$ \\ ${ }^{1}$ Universidade Federal de Uberlândia - Campus Umuarama - Uberlândia, MG \\ ${ }^{2}$ Associação Nacional de Criadores e Pesquisadores - Ribeirão Preto, SP
}

\begin{abstract}
RESUMO
Objetivou-se avaliar a influência da qualidade da informação na predição de valores genéticos para características de crescimento em bovinos da raça Nelore. Foram utilizadas informações de fazendas participantes do Programa Nelore Brasil da Associação Nacional de Criadores e Pesquisadores, considerando-se dados zootécnicos obtidos entre 2012 e 2013. Foram analisadas as características de crescimento relacionadas aos pesos aos 120 (P120), 210 (P210), 365 (P365) e 450 (P450) dias de idade, sob diferentes cenários: inclusão de todas as informações de lote de manejo (cenário de referência) e inclusão aleatória de $90 \%, 70 \%, 50 \%, 30 \%$ e $0 \%$ das informações de lote de manejo dos animais com medidas fenotípicas. Os valores genéticos foram preditos mediante análises unicaracterísticas sob modelo animal. Ocorreram alterações em todos os parâmetros genéticos quando comparados aos obtidos pelo cenário de referência. Houve um aumento nas estimativas de herdabilidade à medida que se reduziu o número de informações sobre os lotes de manejo. Esses resultados sugerem que o progresso genético estimado para rebanhos com baixa qualidade da informação zootécnica pode não expressar adequadamente o que realmente está ocorrendo com o rebanho, uma vez que as estimativas de herdabilidade podem estar infladas. Verificou-se que houve alteração na classificação dos animais para todas as características de crescimento avaliadas. A qualidade da informação zootécnica influencia na predição dos valores genéticos para as características de crescimento.
\end{abstract}

Palavras-chave: bovino de corte, dados zootécnicos, lotes de manejo, parâmetros genéticos

\begin{abstract}
The objective was to evaluate the influence of information quality in the prediction of genetic values for the Nellore cattle's growth traits. The information came from cattle farms participating in the program of the Brazilian National Association of Breeders and Researchers (ANCP), from 2012 to 2013. Field data such as batch or management group were considered assessment criteria, and the growth traits related to body weight at 120 (W120), 210 (W210), 365 (W365) and 450 (W450) days under different scenarios: inclusion of all management lot information; and random inclusion of 90\%, 70\%, 50\%, 30\% and $0 \%$ of the batch management of information from animals with phenotypic measurements. Breeding values were predicted by using univariate models in animal model. Changes on all genetic parameters were compared to those obtained in the reference scenario. There was an increase in the estimates of heritability as it reduced the number of details about lots of management. These results suggest that gene progress estimated herds with low quality information do not adequately represent what truly occurs with the herd, since heritability estimates may be inflated. Change in the classification of animals was found at all the growth traits. The quality information influences the prediction of breeding values for growth traits.
\end{abstract}

Keywords: beef cattle, genetic parameters, management groups, production data

Recebido em 14 de abril de 2016

Aceito em 8 de julho de 2016

*Autor para correspondência (corresponding author)

E-mail: carina@ufu.br 


\section{INTRODUÇÃO}

O agronegócio representa a principal atividade econômica no Brasil, com destaque para o setor de pecuária de corte que, atualmente, ocupa o terceiro lugar em importância (Mapa, 2013).

A pecuária de corte encontra-se em processo de transformação em virtude do aumento do uso de novas tecnologias agropecuárias. Fatores ligados ao manejo e à alimentação dos animais foram decisivos para o crescimento do setor da produção de corte, no entanto muito desse ganho se deve, principalmente, à melhoria contínua do potencial genético dos animais (Faria et al., 2009).

A identificação e a utilização dos melhores genótipos podem promover o progresso genético dos rebanhos. Entretanto, para a identificação de genótipos superiores, é importante considerar informações que realmente expressem a qualidade genética dos indivíduos. Assim, os resultados de qualquer processo de seleção dependem da predição dos valores genéticos dos animais e que serão transmitidos às progênies (Faria et al., 2007).

Predizer o valor genético com precisão é tarefa complicada, no entanto há metodologias que permitem obter essa informação. Conforme descrito por Ferraz e Eler (1999), a predição do valor genético de um animal irá depender da estimativa de herdabilidade da característica em estudo, do número e da qualidade das informações fenotípicas e ambientais e do parentesco entre os animais avaliados.

Por meio de métodos estatísticos, é possível separar os fatores ambientais dos fatores genéticos que contribuem para o desempenho do animal, ou seja, o fenótipo. Entretanto, faz-se necessária a obtenção cuidadosa das informações zootécnicas, pois estas serão interpretadas e utilizadas em modelos de análises genéticas.

Nesse contexto, a correta escrituração zootécnica é de grande importância na predição dos valores genéticos dos animais. Fatores não genéticos, tais como alimentação, ano e estação de nascimento, fazenda, lote de manejo, sexo e idade da vaca ao parto, são informações que podem afetar os resultados das avaliações genéticas (Ugarte et al., 1992; Sarmento et al., 2003).
Em geral, para a realização de uma avaliação genética, os dados coletados nas fazendas (sistemas de manejo ou alimentação) são enviados aos programas de melhoramento genético por meio de sistemas computacionais, sendo interpretados e estruturados para composição do banco de dados. Posteriormente, as análises genéticas são realizadas e os resultados são retornados ao criador.

Atualmente, os programas de melhoramento genético possuem estruturas de coleta de informações zootécnicas, porém essas informações são fornecidas pelos criadores. As falhas na informação de campo podem resultar em avaliações genéticas subjetivas. Conforme descrito por Carneiro et al. (2001), a avaliação genética com alta confiabilidade é fator crucial para uma seleção eficiente.

Cobuci et al. (2006) salientaram que, se os dados de campo (zootécnicos) não forem informados corretamente, podem ocorrer alterações que comprometerão a credibilidade das avaliações genéticas. De acordo com Oliveira (1996), mesmo que sejam utilizadas as mais modernas tecnologias de avaliação genética, o ponto crítico ainda está na qualidade dos dados de campo informados e na formação dos grupos de animais contemporâneos.

Em vista disso, neste trabalho, objetivou-se avaliar a influência da qualidade da informação na predição de valores genéticos para as características de crescimento em bovinos da raça Nelore.

\section{MATERIAL E MÉTODOS}

Para o presente estudo, consideraram-se informações, obtidas nos anos de 2012 a 2013, de bovinos da raça Nelore, pertencentes a cinco fazendas participantes do Programa Nelore Brasil da Associação Nacional de Criadores e Pesquisadores (ANCP). Vale ressaltar que tais fazendas passaram por auditoria quanto à qualidade da informação zootécnica.

Para verificação da importância da qualidade da informação dos dados de campo, considerou-se o lote de manejo como critério de avaliação. O lote de manejo representa os animais que foram submetidos às mesmas condições de ambiente na fazenda. Foram analisadas as características de crescimento relacionadas aos pesos padronizados 
aos 120 (P120), 210 (P210), 365 (P365) e 450 (P450) dias de idade.

Com o intuito de avaliar a importância da informação correta de lotes de manejo na estimação de parâmetros genéticos e na predição dos valores genéticos, consideraram-se os seguintes cenários: (a) conjunto de dados com a inclusão de todas as informações de campo, coletadas adequadamente, e descrição do lote de manejo dos animais que possuem mensurações fenotípicas (REF); (b) conjunto de dados com inclusão aleatória de $90 \%$ das informações do lote de manejo (90LM); (c) conjunto de dados com inclusão aleatória de $70 \%$ das informações do lote de manejo (70LM); (d) conjunto de dados com inclusão aleatória de $50 \%$ das informações do lote de manejo (50LM); (e) conjunto de dados com inclusão aleatória de $30 \%$ das informações do lote de manejo (30LM); e (f) conjunto de dados sem a informação do lote de manejo dos animais com medidas fenotípicas (0LM).

A estruturação dos arquivos de dados foi realizada com a utilização do programa Statistical Analysis System (Statistical..., 2004). A definição dos grupos de animais contemporâneos para verificação das características de crescimento considerou: (i) fazenda, ano e estação de nascimento, sexo e lote de manejo aos 120 dias para P120; (ii) fazenda, ano e estação de nascimento, sexo, regime alimentar e lote de manejo aos 120 e 210 dias para P210; (iii) fazenda, ano e estação de nascimento, sexo, regime alimentar e lote de manejo aos 120, 210 e 365 dias para P365; e (iv) fazenda, ano e estação de nascimento, sexo, regime alimentar e lote de manejo aos 120, 210, 365 e 450 dias para P450. O cenário 0LM não inclui o lote de manejo na formação dos grupos contemporâneos, em virtude da ausência dessa informação.

O efeito de estação de nascimento foi dividido em quatro classes: animais nascidos nos meses de agosto a outubro (classe 1), novembro a janeiro (classe 2), fevereiro a abril (classe 3) e maio a julho (classe 4). Eliminaram-se as informações fenotípicas dos animais que apresentaram valores fenotípicos superiores ou inferiores a 3,5 desvios padrão somados ao valor médio do seu grupo de animais contemporâneos.

No banco de dados, foram obtidas as seguintes informações: os animais com peso aos 120 dias de idade (P120) eram filhos de 132 touros e 9072 vacas; os animais com peso aos 210 dias de idade (P210) eram filhos de 104 touros e 7664 vacas; os animais com P365 eram filhos de 102 touros e 6533 vacas, e os animais com P465 eram filhos de 89 touros e 3887 vacas.

Os parâmetros genéticos das características de crescimento foram estimados mediante análises unicaracterísticas sob modelo animal, utilizandose a estatística bayesiana por meio do aplicativo MTGSAM (Multiple Trait Gibbs Sampler for Animal Models), desenvolvido por Van Tassell et al. (1998). Consideraram-se o grupo de animais contemporâneos (GC) e a classe de idade da vaca ao parto (IVP) como efeitos aleatórios de variação ambiental. $\mathrm{O}$ modelo completo pode ser representado em notação matricial, como:

$$
y=X \beta+Z_{1} a+Z_{2} m+e
$$

em que: ${ }^{y}$ é o vetor das observações, $\beta$ é o vetor dos efeitos aleatórios de ambiente, $a$ é o vetor dos efeitos aleatórios que representam os valores genéticos aditivos diretos de cada animal, $m$ é o vetor dos efeitos aleatórios que representam os valores genéticos aditivos maternos de cada animal, $e$ é o vetor de efeitos aleatórios residuais, e $X, Z_{1}$ e $Z_{2}$ são as matrizes de incidência que relacionam as observações aos efeitos aleatórios de ambiente e aos efeitos aleatórios genéticos aditivos direto e materno, respectivamente. Ressalta-se que o efeito aleatório genético aditivo materno foi incluído somente nas análises para os pesos aos 120 (120) e 210 (P210) dias de idade; já o efeito de ambiente permanente materno não foi considerado no modelo por não haver fêmeas com mais de um filho no banco de dados (medidas repetidas).

O arquivo de genealogia foi composto por 30.125 animais, com consanguinidade média de $2,7 \%$, totalizando 10.235 animais com algum grau de endogamia. Na aplicação do amostrador de Gibbs, foi utilizado um tamanho de cadeia inicial de 300.000 ciclos, sendo os primeiros 50.000 ciclos descartados e as amostras retiradas a cada 1.000 ciclos, totalizando 250 amostras de componentes de variância e parâmetros genéticos. As análises das amostras, da correlação serial e da convergência da cadeia de Gibbs foram realizadas com o auxílio do 
programa GIBANAL (Van Kaam, 1998). O parâmetro v é o grau de liberdade correspondente à distribuição Wishart invertida, indicando o grau de confiabilidade da distribuição inicial. Neste estudo, o parâmetro v utilizado foi de valor zero, ou seja, não refletia nenhum grau de conhecimento sobre os parâmetros.

Para verificar possíveis alterações na classificação dos animais, mediante a comparação dos valores genéticos obtidos com base nas informações corretas (conjunto de dados de referência) e daqueles obtidos sem adequada qualidade de informação, utilizou-se a correlação de posto ou Spearman (Statistical..., 2004).

\section{RESULTADOS E DISCUSSÃO}

Ao se avaliar a estrutura de dados (Tab. 1), verifica-se que praticamente não houve mudanças nos valores médios fenotípicos para todas as características de crescimento, bem como nas estimativas de desvio-padrão e coeficiente de variação dos dados avaliados, considerando-se os diferentes cenários de inclusão de informações de lotes de manejo comparado ao conjunto de dados de referência (REF).

Entretanto, houve alterações no número de animais com mensurações fenotípicas para as características de crescimento avaliadas devido ao tratamento estatístico realizado no banco de dados antes do procedimento para avaliação genética. Conforme descrito, são eliminados os valores fenotípicos considerados outliers dentro do grupo contemporâneo. Assim, os valores fenotípicos da característica avaliada, com valores de 3,5 desvios padrão, acima ou abaixo da média do seu grupo contemporâneo, são eliminados. De acordo com Yamaki (2009), essa restrição na estruturação de arquivos de dados pode influenciar na avaliação genética de uma população.

Tabela 1. Estatística descritiva para características de crescimento, avaliadas em bovinos da raça Nelore, considerando-se os diferentes cenários de inclusão das informações de lotes de manejo

\begin{tabular}{|c|c|c|c|c|c|}
\hline Característica & $\mathrm{N}^{\mathbf{0}}$ animais & Média (kg) & DP (kg) & $\mathrm{CV}(\%)$ & $\mathrm{N}^{\circ} \mathrm{GC}$ \\
\hline \multicolumn{6}{|l|}{ REF } \\
\hline P120 & 10.045 & 134 & 19 & 14 & 564 \\
\hline P210 & 7.672 & 198 & 28 & 14 & 537 \\
\hline P365 & 6.533 & 247 & 41 & 16 & 710 \\
\hline P450 & 3.890 & 287 & 50 & 17 & 460 \\
\hline \multicolumn{6}{|l|}{ 90LM } \\
\hline P120 & 10.033 & 133 & 19 & 14 & 593 \\
\hline P210 & 7.532 & 200 & 28 & 14 & 646 \\
\hline P365 & 6.252 & 248 & 41 & 16 & 826 \\
\hline P450 & 3.647 & 288 & 50 & 17 & 530 \\
\hline \multicolumn{6}{|l|}{ 70LM } \\
\hline P120 & 10.009 & 133 & 19 & 14 & 537 \\
\hline P210 & 7.498 & 200 & 28 & 14 & 664 \\
\hline P365 & 6.124 & 247 & 41 & 17 & 868 \\
\hline P450 & 3.405 & 286 & 52 & 18 & 533 \\
\hline \multicolumn{6}{|l|}{ 50LM } \\
\hline P120 & 9.991 & 133 & 19 & 14 & 454 \\
\hline P210 & 7.500 & 198 & 28 & 14 & 589 \\
\hline P365 & 6.125 & 246 & 41 & 18 & 788 \\
\hline P450 & 3.449 & 285 & 51 & 18 & 513 \\
\hline \multicolumn{6}{|l|}{ 30LM } \\
\hline P120 & 10.008 & 133 & 19 & 14 & 382 \\
\hline P210 & 7.526 & 198 & 28 & 14 & 467 \\
\hline P365 & 6.198 & 245 & 40 & 17 & 570 \\
\hline P450 & 3.570 & 284 & 50 & 18 & 386 \\
\hline \multicolumn{6}{|l|}{ OLM } \\
\hline P120 & 10.113 & 134 & 19 & 14 & 66 \\
\hline P210 & 7.784 & 198 & 28 & 14 & 65 \\
\hline P365 & 6.763 & 247 & 41 & 16 & 69 \\
\hline P450 & 4.076 & 287 & 50 & 17 & 46 \\
\hline
\end{tabular}

DP: desvio-padrão, CV: coeficiente de variação; GC: grupo contemporâneo. 
Em relação ao grupo de animais contemporâneos (GC), verificou-se que, à medida que se alterou o número de informações sobre os lotes de manejo, ocorreram grandes mudanças no número de grupos contemporâneos para as características de crescimento avaliadas (Tab. 1).

Essas mudanças na quantidade de grupos contemporâneos são verificadas, principalmente, no cenário 0LM, em que não foram incluídas as informações de lotes de manejo no banco de dados para as características de crescimento avaliadas (Tab. 1). Nessa condição, os grupos contemporâneos apresentaram-se em menor número, porém com maior quantidade de animais em cada GC.

Apesar de Oliveira (1996) ressaltar que quanto maior o número de animais em um mesmo grupo contemporâneo, maior será a confiabilidade nas predições das DEPs (diferença esperada na progênie), observou-se que, no presente estudo, o aumento do número de animais por nível de efeito fixo (grupo contemporâneo) foi reflexo da pouca qualidade da informação dos efeitos ambientais.

Em relação às estimativas de componentes de (co)variância e aos parâmetros genéticos, verificou-se que houve alterações em todas as estimativas, considerando-se os diferentes cenários de inclusão das informações de lotes de manejo comparados ao cenário de referência (Tab. 2). Observa-se que houve um aumento nas estimativas de herdabilidade para todas as características de crescimento à medida que se reduziu o número de informações sobre os lotes de manejo, exceto para os cenários 10LM e 30LM, que apresentaram poucas alterações entre si, embora com estimativas de herdabilidade inferiores ao cenário de referência (REF).

Ao avaliar a variância genética aditiva para todas as características de crescimento, observa-se que ela aumentou quando se reduziu o número de informações sobre os lotes de manejo; assim, pode-se inferir que houve desvio de parte da variação do componente ambiental para o componente relacionado aos efeitos genéticos, o que provocou uma superestimação. Esses resultados corroboram os relatos de Ugarte et al. (1992), Oliveira (1996) e Cobuci et al. (2006), que inferiram que a baixa qualidade da informação zootécnica pode gerar predições genéticas com baixa confiabilidade.

Esses resultados observados neste estudo também sugerem que o progresso genético estimado para os rebanhos que apresentam baixa qualidade da informação zootécnica pode não expressar adequadamente o que realmente está ocorrendo com a população, uma vez que as estimativas de herdabilidade podem estar infladas. De acordo com Yamaki (2009), as estratégias de avaliação devem garantir a estimação acurada dos parâmetros genéticos das populações. Ferraz e Eler (1999) afirmaram que equívocos na constituição de grupos contemporâneos podem levar os melhoristas a ignorarem as diferenças de lotes de manejo, gerando valores genéticos superestimados para os animais que foram criados em melhores condições ambientais.

A estatística descritiva dos valores genéticos para as características de crescimento (pesos), considerando-se os diferentes cenários de inclusão das informações de lote de manejo, é descrita na Tab. 3.

Observa-se que os valores com maior discrepância foram aqueles obtidos no cenário sem a inclusão da informação dos lotes de manejo para composição dos grupos de animais contemporâneos (Tab. 3). Essa maior variabilidade evidencia a superestimação e a subestimação dos valores genéticos dos animais avaliados no cenário 0LM. Na ausência de informações sobre os lotes de manejo (0LM), os grupos contemporâneos sofreram alterações na sua constituição, e, consequentemente, os valores genéticos preditos para as características de crescimento também se alteraram, gerando valores genéticos superestimados para os animais criados em melhores condições ambientais e valores genéticos subestimados para os que foram criados em piores condições de ambiente. 
Tabela 2. Estimativas médias dos componentes de (co)variância e parâmetros genéticos para as características de crescimento de bovinos da raça Nelore, considerando-se os diferentes cenários de inclusão das informação de lotes de manejo

\begin{tabular}{|c|c|c|c|c|c|c|}
\hline \multirow{2}{*}{ Parâmetro genético } & \multicolumn{6}{|c|}{ Cenários } \\
\hline & REF & 90LM & $70 \mathrm{LM}$ & 50LM & $30 \mathrm{LM}$ & OLM \\
\hline \multicolumn{7}{|l|}{ P120 } \\
\hline$\sigma_{a}^{2}$ & 24,76 & 13,19 & 8,70 & 14,71 & 27,32 & 72,03 \\
\hline$\sigma_{\mathrm{am}}$ & $-5,87$ & $-0,18$ & $-2,58$ & $-10,69$ & $-7,68$ & $-25,82$ \\
\hline$\sigma_{m}^{2}$ & 43,16 & 18,92 & 12,95 & 18,52 & 16,27 & 63,23 \\
\hline$\sigma_{r}^{2}$ & 153,02 & 117,60 & 93,81 & 88,24 & 93,93 & 143,37 \\
\hline $\mathrm{h}_{\mathrm{a}}^{\mathrm{I}^{2}}$ & 0,11 & 0,08 & 0,07 & 0,13 & 0,21 & 0,28 \\
\hline $\mathrm{h}_{\mathrm{m}}^{2}$ & 0,20 & 0,12 & 0,11 & 0,16 & 0,12 & 0,24 \\
\hline $\mathrm{r}_{\mathrm{am}}$ & $-0,16$ & 0,02 & $-0,21$ & $-0,63$ & $-0,34$ & $-0,37$ \\
\hline
\end{tabular}

\section{P210}

$\sigma_{\mathrm{a}}^{2}$

$\sigma_{\mathrm{am}}$

$\sigma_{2}^{2}$

$\sigma_{r}^{2}$

$\mathrm{h}_{\mathrm{a}}^{2}$

$\mathrm{h}_{\mathrm{m}}^{2}$

$\mathrm{r}_{\mathrm{am}}$

$\begin{array}{rr}73,25 & 39,93 \\ -23,86 & -16,93 \\ 59,48 & 21,38 \\ 289,29 & 225,89 \\ 0,18 & 0,14 \\ 0,14 & 0,07 \\ -0,33 & -0,57\end{array}$

25,49
2,27
9,96
170,47
0,12
0,04
0,39

48,14
$-5,73$
2,36
156,05
0,23
0,01
$-0,57$

86,88

187,42

$-11,56 \quad-60,37$

$7,75 \quad 75,26$

$167,31 \quad 320,44$

\section{P365}

\begin{tabular}{lrrrrrr}
$\sigma_{\mathrm{a}}^{2}$ & 94,07 & 45,30 & 32,78 & 36,81 & 77,04 & 205,87 \\
$\sigma_{\mathrm{r}}^{2}$ & 408,93 & 309,05 & 232,28 & 224,32 & 258,95 & 503,72 \\
$\mathrm{~h}^{2}$ & 0,18 & 0,12 & 0,12 & 0,14 & 0,22 & 0,28 \\
& & & & & \\
$\mathbf{P 4 5 0}$ & & & & & & \\
$\sigma_{\mathrm{a}}^{2}$ & 148,92 & 71,92 & 54,92 & 124,01 & 234,53 & 419,11 \\
$\sigma_{\mathrm{r}}^{2}$ & 453,30 & 362,91 & 286,04 & 202,11 & 183,97 & 430,94 \\
$\mathrm{~h}^{2}$ & 0,24 & 0,16 & 0,16 & 0,37 & 0,55 & 0,49 \\
\hline$\sigma^{2}{ }_{\mathrm{a}}^{2}$ : variância genética aditiva direta; $\sigma_{\mathrm{am}}:$ covariância entre os efeitos aditivos direto e materno; $\sigma_{\mathrm{m}}^{2}:$ variância
\end{tabular} $\sigma_{\mathrm{a}}^{2}$ : variância genética aditiva direta; $\sigma_{\mathrm{am}}$ : covariância entre os efeitos aditivos direto e materno; $\sigma_{\mathrm{m}}^{2}$ : variância
genética aditiva materna; $\sigma_{\mathrm{r}}^{2}$ : variância residual; $\mathrm{h}^{2}$ a coeficiente de herdabilidade para os efeitos genéticos aditivos diretos; $\mathrm{h}_{\mathrm{m}}^{2}$ : herdabilidade do efeito materno; $\mathrm{r}_{\mathrm{am}}$ : correlação genética aditiva entre os efeitos direto e materno.

Por fim, observa-se, na Tab. 3, que, mesmo nos cenários em que somente $10 \%$ (10LM) ou $30 \%$ (30LM) dos animais não apresentaram informações de lotes de manejo, houve alterações nas estimativas de médias dos valores genéticos preditos para todas as características de crescimento avaliadas. Dessa forma, pode-se inferir que qualquer equívoco na informação, como, por exemplo, dos lotes de manejo, interfere na predição dos valores genéticos e na correta identificação dos animais geneticamente superiores. Resultados semelhantes foram relatados por Cobuci et al. (2006).

$\mathrm{Na}$ Tab. 4, são apresentadas as correlações de posto ou Spearman dos valores genéticos obtidos com base em dados com qualidade na informação (cenário REF), comparados aos obtidos nos diferentes cenários em que foram excluídas informações de lotes de manejo em diferentes proporções. Verifica-se que houve alteração na classificação dos animais para todas as características de crescimento avaliadas, em três grupos de acurácia dos valores genéticos.

Esses resultados corroboram a conclusão a que chegaram Ferraz e Eler (1999) em seu estudo: as falhas de informações nos lotes de manejo influenciam na formação dos grupos de contemporâneos e, consequentemente, causam alterações na avaliação genética dos animais. Quando os lotes de manejo não são devidamente informados, há o risco de ocorrer uma seleção equivocada, uma vez que a alteração de classificação pode favorecer animais que talvez não apresentem o potencial genético esperado. 
A importância da qualidade...

Tabela 3. Médias dos valores genéticos preditos (diretos e maternos) para as características de crescimento, avaliadas em bovinos da raça Nelore, considerando-se os diferentes cenários de inclusão da informação dos lotes de manejo

\begin{tabular}{|c|c|c|c|c|c|c|}
\hline Valores Genéticos & REF & 90LM & $70 \mathrm{LM}$ & $50 \mathrm{LM}$ & $30 \mathrm{LM}$ & $0 \mathrm{LM}$ \\
\hline \multicolumn{7}{|l|}{ Médias } \\
\hline DP120 & 0,85 & 0,48 & 0,45 & 0,73 & 1,50 & 3,26 \\
\hline MP120 & 0,61 & 0,40 & $-0,01$ & $-0,35$ & $-0,18$ & $-0,87$ \\
\hline DP210 & 1,84 & 0,92 & 0,69 & 1,60 & 3,09 & 6,36 \\
\hline MP210 & 0,09 & $-0,21$ & 0,09 & $-0,18$ & $-0,36$ & $-1,78$ \\
\hline P365 & 2,14 & 0,80 & 0,74 & 1,14 & 2,50 & 5,77 \\
\hline P450 & 3,56 & 1,62 & 1,58 & 2,88 & 5,87 & 10,24 \\
\hline \multicolumn{7}{|l|}{ Desvio-padrão } \\
\hline DP120 & 1,80 & 1,27 & 1,01 & 1,22 & 2,32 & 4,79 \\
\hline MP120 & 2,61 & 1,58 & 1,12 & 1,37 & 1,11 & 2,93 \\
\hline DP210 & 3,46 & 2,27 & 1,99 & 2,89 & 4,69 & 8,74 \\
\hline MP210 & 2,14 & 1,09 & 0,73 & 0,30 & 0,50 & 2,91 \\
\hline P365 & 4,37 & 2,59 & 2,06 & 1,99 & 3,75 & 9,16 \\
\hline P450 & 5,65 & 3,28 & 2,75 & 4,45 & 7,95 & 14,09 \\
\hline \multicolumn{7}{|l|}{ Valor mínimo } \\
\hline DP120 & $-6,24$ & $-3,85$ & $-2,33$ & $-5,31$ & $-8,34$ & $-12,26$ \\
\hline MP120 & $-13,58$ & $-6,17$ & $-5,77$ & $-8,82$ & $-7,45$ & $-18,97$ \\
\hline DP210 & $-13,32$ & $-6,25$ & $-7,20$ & $-13,85$ & $-23,55$ & $-26,30$ \\
\hline MP210 & $-14,67$ & $-13,20$ & $-3,63$ & $-2,65$ & $-2,97$ & $-22,79$ \\
\hline P365 & $-14,04$ & $-8,20$ & $-7,19$ & $-9,52$ & $-16,16$ & $-27,12$ \\
\hline P450 & $-22,46$ & $-9,35$ & $-11,16$ & $-25,32$ & $-41,74$ & $-47,44$ \\
\hline \multicolumn{7}{|l|}{ Valor máximo } \\
\hline DP120 & 18,36 & 11,86 & 11,14 & 9,81 & 13,26 & 34,61 \\
\hline MP120 & 14,82 & 9,90 & 6,69 & 7,19 & 5,53 & 16,75 \\
\hline DP210 & 41,52 & 30,47 & 21,86 & 23,93 & 25,18 & 70,49 \\
\hline MP210 & 11,04 & 4,16 & 3,98 & 1,39 & 2,22 & 8,45 \\
\hline P365 & 44,05 & 31,08 & 25,41 & 18,06 & 22,44 & 81,52 \\
\hline P450 & 54,18 & 39,24 & 35,19 & 39,05 & 48,73 & 100,88 \\
\hline
\end{tabular}

DP120: valor genético aditivo direto do P120; MP120: valor genético aditivo materno do P120; DP210: valor genético aditivo direto do P210; MP120: valor genético aditivo materno do P210.

Verifica-se também que, em geral, os valores da correlação de posto ou Spearman foram mais baixos nos cenários 50LM e 30LM, indicando que, nessas condições, as predições dos valores genéticos são mais prejudicadas do que na ausência completa da informação de lotes de manejo.

Em relação aos valores genéticos aditivos maternos, a influência da alteração na qualidade da informação foi evidenciada pelas grandes mudanças na classificação dos animais, comparados aos valores genéticos obtidos para todas as características avaliadas, com banco de dados de qualidade (cenário REF). Esse resultado mostra que o efeito maternal é fortemente influenciado pela baixa qualidade na informação sobre os lotes de manejo.
São incontestáveis as demandas por qualidade do mercado mundial. Para alcançar o desejado padrão de qualidade, os teóricos da administração chamam a atenção para a adequada gestão dos processos e dos recursos humanos, principalmente o pessoal envolvido com a coleta de dados e a tecnologia da informação. Os resultados apresentados neste estudo demonstraram que erros na coleta de dados poderão levar o empresário rural à tomada de decisões que não contribuirão para o desenvolvimento de seu negócio. $\mathrm{Na}$ era do conhecimento, em que a informação, por si só, não diz nada, se a coleta de dados não for feita de forma confiável, os modelos matemáticos podem produzir informações equivocadas. 
Tabela 4. Correlação de posto ou Spearman entre os valores genéticos preditos para as características de crescimento, avaliadas em bovinos da raça Nelore, considerando-se o cenário de referência (REF), em três grupos de acurácia dos valores genéticos, com aqueles preditos em diferentes níveis de inclusão das informações de lotes de manejo

\begin{tabular}{|c|c|c|c|c|c|}
\hline & 90LM & 70LM & 50LM & 30LM & OLM \\
\hline \multicolumn{6}{|c|}{ Valores genéticos com acurácia $\leq \mathbf{3 0} \%$} \\
\hline P120 & 0,82 & 0,75 & 0,43 & 0,74 & 0,87 \\
\hline MP120 & 0,83 & 0,64 & 0,36 & 0,55 & 0,69 \\
\hline P210 & 0,90 & 0,64 & 0,78 & 0,79 & 0,91 \\
\hline MP210 & 0,57 & 0,57 & 0,02 & 0,19 & 0,45 \\
\hline P365 & 0,71 & 0,71 & 0,64 & 0,64 & 0,81 \\
\hline $\mathrm{P} 450$ & 0,81 & 0,77 & 0,74 & 0,71 & 0,81 \\
\hline \multicolumn{6}{|c|}{ Valores genéticos com acurácia entre $31 \%$ e $69 \%$} \\
\hline P120 & 0,90 & 0,80 & 0,74 & 0,77 & 0,86 \\
\hline MP120 & 0,91 & 0,80 & 0,58 & 0,64 & 0,75 \\
\hline $\mathrm{P} 210$ & 0,90 & 0,79 & 0,76 & 0,77 & 0,88 \\
\hline MP210 & 0,82 & 0,68 & 0,02 & 0,29 & 0,63 \\
\hline P365 & 0,88 & 0,82 & 0,68 & 0,72 & 0,88 \\
\hline $\mathrm{P} 450$ & 0,92 & 0,76 & 0,59 & 0,58 & 0,74 \\
\hline \multicolumn{6}{|c|}{ Valores genéticos com acurácia $\geq 70 \%$} \\
\hline P120 & 0,89 & 0,76 & 0,68 & 0,79 & 0,87 \\
\hline MP120 & 0,93 & 0,88 & 0,81 & 0,81 & 0,88 \\
\hline $\mathrm{P} 210$ & 0,95 & 0,85 & 0,63 & 0,63 & 0,88 \\
\hline MP210 & 0,83 & 0,75 & 0,14 & 0,72 & 0,92 \\
\hline P365 & 0,93 & 0,84 & 0,59 & 0,57 & 0,85 \\
\hline $\mathrm{P} 450$ & 0,94 & 0,55 & 0,42 & 0,40 & 0,47 \\
\hline
\end{tabular}

Para garantir a aplicabilidade e a eficácia da qualidade da informação, o treinamento técnico realizado por meio de ferramentas necessárias, estatística, entre outros, deve se integrar ao estudo da origem, do conceito, dos princípios e dos fundamentos da qualidade como ciência (Barros, 1993). No caso da coleta de dados de campo, o processo deverá ser não só mapeado, mas alinhado ao contexto geral tanto da economia nacional quanto da mundial. Não basta mapear o processo e controlá-lo, é necessário também divulgá-lo para toda a cadeia de usuários, principalmente para os funcionários envolvidos diretamente na coleta, a fim de que compreendam, por exemplo, a importância de informar, com critérios claros, o lote de manejo a que o animal foi submetido, tendo em vista todas as alterações demonstradas na presente pesquisa.

\section{CONCLUSÕES}

A qualidade da informação zootécnica influencia na predição dos valores genéticos para as características de crescimento de bovinos da raça Nelore. Essa influência provoca alterações na classificação dos animais, o que pode levar à redução do ganho genético dos rebanhos, devido ao uso de animais erroneamente identificados como melhoradores. Recomenda-se atenção quanto ao fornecimento da informação correta dos lotes de manejo aos programas de melhoramento genético, para a obtenção de predições acuradas dos valores genéticos para as características de interesse.

\section{REFERÊNCIAS}

BARROS, C.D.C. Sensibilizando para a qualidade. Rio de Janeiro: Qualitymark, 1993. $95 \mathrm{p}$.

BRASIL. Ministério da Agricultura, Pecuária e Abastecimento. Assessoria de Gestão Estratégica. Projeções do agronegócio - 2012/13 a 2022/23. 96p. 2013. Disponível em: <http://www.agricultura.gov.br>. Acessado em: 07 Jun. 2014.

CARNEIRO, A.P.S.; TORRES, R.A.; EUCLYDES, R.F. et al. Efeito da conexidade de dados sobre o valor fenotípico médio e a variância genética aditiva. Rev. Bras. Zootec., v.30, p.336-341, 2001. 
COBUCI, J.A.; ABREU, U.G.P.; TORRES, R.A. Formação de grupos contemporâneos em bovinos de corte. 21.ed. Corumbá: Embrapa Pantanal, 2006. Disponível em: <27phttp://www.cpap.embrapa.br/publicacoes/on line/DOC87.pdf.>. Acessado em: 20 mar. 2013.

FARIA, C.U.; MAGNABOSCO, C.U.; ALBUQUERQUE, L.G. et al. Bayesian analysis of twinning and ovulation rates using a multipletrait threshold model and Gibbs sampling. Arq. Bras. Med. Vet. Zootec., v.61, p.949-958, 2009.

FARIA, C.U.; MAGNABOSCO, C.U.; REYES, A.L. et al. Bayesian inference in a quantitative genetic study of growth traits in Nelore cattle (Bos indicus). Genet. Mol. Biol, v.30, p.545-551, 2007.

FERRAZ, S.B.J.; ELER, P.J. Seleção de zebuínos para características produtivas. In: SIMPÓSIO DE PRODUÇÃO DE GADO DE CORTE, GRUPO DE MELHORAMENTO ANIMAL UNIVERSIDADE DE SÃO PAULO, 1999, São Paulo. Anais... São Paulo: Faculdade de Zootecnia e Engenharia de Alimentos, Pirassununga, SP, 1999. 22p.

OLIVEIRA, H.N. Grupos de contemporâneos e conectabilidade. In: CURSO SOBRE AVALIAÇÃO GENÉTICA DE BOVINOS DE CORTE EM GOIÁS, 1996, Goiânia. Anais... Goiânia: Núcleo Regional de Goiânia do Programa de Melhoramento Genético da Raça Nelore, 1996. p.39-50.
SARMENTO, J.L.R.; PIMENTA FILHO, E.C.; RIBEIRO, M.N. et al. Efeitos ambientais e genéticos sobre o ganho em peso diário de bovinos Nelore no estado da Paraíba. Rev. Bras. Zootec., v.32, p.325-330, 2003.

STATISTICAL Analysis System. Version 9.1.3. Cary: SAS Institute, 2004.

UGARTE, E.; ALENDA, R.; CARABANO, M.J. Fixed and random contemporary groups in genetic evaluations. J. Dairy Sci., v.75, p.269278, 1992.

VAN KAAM J.B.C.H.M. Gibanal 2.9: analyzing program for Markov chain Monte Carlo sequences. Wageningen: Department Animal Science, Wageningen Agricultural University, 1998.

VAN TASSELL, C.P.; VAN VLECK, L.D.; GREGORY, K.E. Bayesian analysis of twinning and ovulation rates using a multiple-trait threshold model and Gibbs sampling. J. Anim. Sci., v.76, p.2048-2061, 1998.

YAMAKI, M. Impacto da restrição de dados na avaliação genética animal. 2009. 46f. Tese (Doutorado) - Departamento de Genética e Melhoramento, Universidade Federal de Viçosa, Viçosa, MG. 